\title{
VIVACE Context Based Search Platform
}

\author{
Romaric Redon ${ }^{1}$, Andreas Larsson ${ }^{2}$, Richard Leblond ${ }^{1}$, and Barthelemy Longueville ${ }^{1}$ \\ ${ }^{1}$ EADS France, Innovation works, TCC5 simulation, IT \& systems engineering, Marius Terce. \\ 18,31300 Toulouse, France \\ \{romaric.redon, richard.leblond, barthelemy.longueville\} deads.net \\ ${ }^{2}$ Faste Laboratory, Luleå University of Technology, 97187 Luleå, Sweden \\ andreas.c.larssonaltu.se
}

\begin{abstract}
One of the key challenges of knowledge management is to provide the right knowledge to the right person at the right time. To face this challenge, a context based search platform was developed in the frame of the European Integrated Project VIVACE. This platform is based on the identification of a user context and the subsequent pushing of applicable knowledge to that particular user. We introduce a context model to represent the user's context. This context model is used to describe the context of an engineer working in a specific company. Further, we developed means to index available knowledge based on company engineering context and means to search for knowledge applicable to the user's context. Since it is not always possible to describe in which context the knowledge assets should be applied, we added learning capabilities which enable the system to learn the applicability of specific knowledge to a user's context based on user feedback.
\end{abstract}

Keywords: Knowledge management, context aware systems and applications, context modelling, analogy and case based reasoning.

\section{Introduction}

This article deals with a presentation of the context based search platform developed in the frame of VIVACE - an Integrated Project within the European Commission's Sixth Framework Programme (FP6). After this introduction Part II provides an outline of the VIVACE project and describes the overall objectives of the Knowledge Enabled Engineering (KEE) Work Package. Part III focuses on KEE modelling activities: knowledge modelling and context modelling. Part IV deals with a detailed description of the concept of context based search and a presentation of the platform capabilities. In Part V the main features of the prototype developed in 2006 are presented as well as early experimentation results. Foreseen short term and longer term perspectives are discussed in part VI. Finally, part VII provides some conclusions.

\section{VIVACE Knowledge Enabled Engineering}

VIVACE [1] is a €70M Integrated Project in the EC Sixth Framework Programme (FP6). The acronym stands for 'Value Improvement through a Virtual Aeronautical 
Collaborative Enterprise', with the main project goal to support the design of a complete aircraft, including engines, by providing increased simulation capabilities throughout the product-engineering life cycle. Briefly stated, the goal is to create a 'virtual product' in a 'virtual enterprise' - thereby aiming to achieve a 5\% cost reduction in aircraft development and a 5\% reduction of the development phase of a new aircraft design combined with a contribution to a $30 \%$ reduction in the lead time and $50 \%$ reduction in development costs respectively for a new or derivative gas turbine engine.

The Knowledge Enabled Engineering (KEE) Work Package is one of six integrated technical packages that collectively form the Advanced Capabilities subproject (SP3) of VIVACE. Starting from the well-known knowledge management phases (storage, retrieval, reuse, sharing etc.), the main goal is to define and exploit advanced methods that could help companies capture how knowledge is used, and allow them to radically improve their engineering process by leveraging past design experience. The Work Package focuses on providing methods, tools, solutions and training activities that create conditions for the successful integration of knowledge enabling solutions both on the business side and on the technical side of the project's existing application environment of aeronautics. Beyond the consortium, the work promotes and facilitates the consolidation of the vision of a consistent way of building a real 'knowledgedriven' virtual enterprise, starting from the assumption that the developed core system will be applicable independent of the individual company type of business.

KEE can be considered as the exploitation of Knowledge Management within an engineering context, which fundamentally means leveraging knowledge sources in order to enable engineers to complete their work quickly and correctly. Thus, KEE is about providing the right information to the engineer, at the right time, in the right format, in a collaborative environment that promotes learning within the organization, across the supply chain and across the Extended Enterprise. Therefore, the KEE Work Package proposed to design a context based search platform that would enable users to search for knowledge which is applicable to their contexts. This article will focus on the description of the platform even though KEE also produce results in other area such as facilitating knowledge sharing, managing lessons learned, assessing team relationships and assessing maturity in a gated decision process.

\section{KEE Modelling Activities}

In order to proceed towards the development of a platform that provide applicable engineering knowledge depending on the user's context, a first required step was to specify what we mean by engineering knowledge and user context. The following paragraphs describe the models we introduced in KEE to represent engineering knowledge and user context.

\subsection{Engineering Knowledge Modelling}

Engineering knowledge deals with knowledge about products, processes and organisations. A key issue is that engineering knowledge is often stored in people's head or diluted with other possibly irrelevant, information in technical documents. 
In order to support proper capture of engineering knowledge, methodologies such as CommonKADS[2] and MOKA[3] were proposed. These methodologies enable the building of knowledge models composed of interlinked Knowledge Elements (K-El). $\mathrm{K}-\mathrm{El}$ are pieces of knowledge focusing on specific topics. MOKA introduces different forms to support the capturing and structuring of knowledge about both product and process. Entity and Constraints forms enable the collection of knowledge about product breakdown and product limitations. Activity and rules forms enable the collection of knowledge about process breakdown and flow control. At last Illustration forms could be linked to any of the other forms so as to record any corresponding past experience.

These forms are an example of how to organize structured K-El. Other examples of $\mathrm{K}-\mathrm{El}$ found in industrial companies may include documents about lessons learnt, best practices, expert manuals or also expert contact information etc.

The smaller the K-El, the more the process of delivery-in-context makes sense. The objective is then to select the right K-El which do apply to the user context. If all K-El are merged in a single big document, and without any possibility to discriminate them, the in-context delivery process will make no sense or will be time consuming. This single big document will be applicable in almost all user contexts, and the individual user will not know (or with delay) which knowledge in the document does really apply to his/her context.

KEE introduces also the concept of Knowledge Source (K-Source). A K-Source is a K-El container. Examples of a K-Source could be a simple file repository for managing K-El which are stand alone documents, a web application for managing $\mathrm{K}$ El which are interlinked web pages, or a complex content management system for managing structured interlinked K-El. A K-source usually provides standard capabilities such as index extraction and search capabilities. For a windows file repository, for example these capabilities are provided through Microsoft index server.

\subsection{Context Modelling}

The objective of this chapter is to describe the work performed in order to represent user context in engineering. The aim was to propose a relevant context model that could easily be understood by engineers and that we can use to quickly develop a platform in order to gain the end-user buy-in.

First of all, it is worth noting that context is still an ill-defined concept such as discussed in [4]. In order to define the context model to use within VIVACE, we investigated two approaches. First a top-down approach which studies existing context models already proposed in the literature and tries to adjust them to fit our needs. Second a bottom-up approach which starts from the study of existing K-El and aims at describing their context of use.

Top-Down Approach. According to Dey et al [5] context is any information that can be used to characterise the situation of an entity. Based on this definition we can propose that engineering context is any information that can be used to characterise the situation of an engineer. 
In the literature different context models were proposed, some of them, developed for context representation of mobile users focus more on describing the user's physical context [6] (i.e. his/her current location, device, available resources, etc.), whereas some others include the description of the user's organizational context (role, group membership, tasks, etc.). In this last category, context description model proposed by Kirsch et al. [7] retain our attention. It is based on five viewpoints: space, tool, time, community and process. Several context representation classes are used to describe each viewpoint as shown in the following UML diagram.

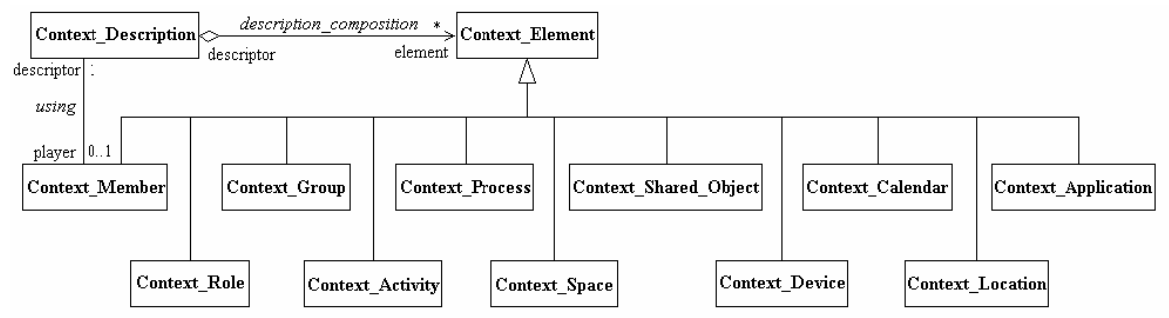

Fig. 1. Kirsch et al. [7] context description model

For KEE use cases, the influence of the user's physical context is not that important: we mainly target engineers working in design offices. Somehow, these engineers always have access to the same resources through the same type of device (i.e. their computer on the network). It means that at a first glance the Context_Space Context_Location, Context_Device representation classes maybe less relevant than the other one for our problem situation.

Bottom-Up Approach. Edmonds [8] says that context is the abstraction of those elements of circumstances in which a model is learnt $[\ldots]$, that allows recognition of new circumstances where the model can be usefully applied. In order to achieve our objective of in-context K-El delivery, we focused on representation of an engineering context, which is the abstraction of those elements of circumstances in which a K-El is learnt $[\ldots]$, that allows recognition of new circumstances where the K-El could be usefully applied. Therefore, we followed recommendations from Longueville et al. [9] to formalize what they call the explicit context. We studied real examples of K-El coming from VIVACE use cases and we identified how to describe their domain of applicability. The result from this bottom-up approach was the identification of relevant context dimensions. Context dimensions are properties or attributes that describe the context. Six context dimensions arose from the analysis: product, activity, project, gate, role and discipline. The domain of applicability for a $\mathrm{K}-\mathrm{El}$ is described by specifying associated context dimension values. For example, a specific K-El may be applicable for the balancing process (activity) of the blade (product), within the scope of X34 (project) during preliminary design (gate) for a simulation engineer (role) in dynamic analysis (discipline). 
We synthesized results coming from both approaches to propose the following UML engineering domain model. Our engineer is identified as a member of an organization; she/he has a role and skills in disciplines and takes part into projects (Community viewpoint). The organization provides her/him with applications running on various devices (Tool viewpoint). she/he performs activities that support product design (Process viewpoint) at a given gate (Time viewpoint) of the project.

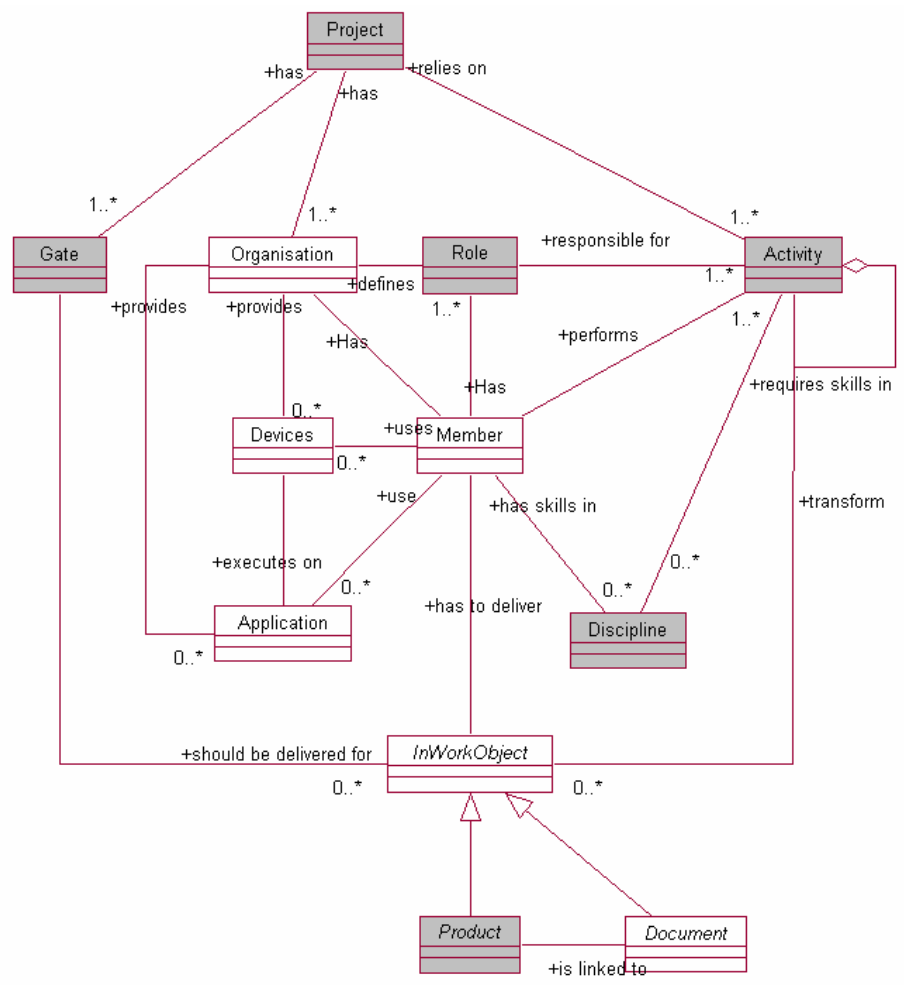

Fig. 2. KEE engineering domain model

According to the results of the bottom-up approach, classes in white were qualified, at a first glance, as less relevant than the other ones for our objective of incontext knowledge delivery. We finally proposed to rely only on six context dimensions to describe the context of our engineer. We introduced context dimension classes to potentially handle complex information associated to each context dimension (e.g. several attributes may be used to describe the product). A simplified context description model with context dimension modelled as attributes was also proposed.

In order to describe a company specific context description model we relied on the simplified context description model and managed the list of possible values for each 
context dimension in form of a tree. For example, the following tree organizes the list of possible values for the product context dimension for the AVIO company context description model.

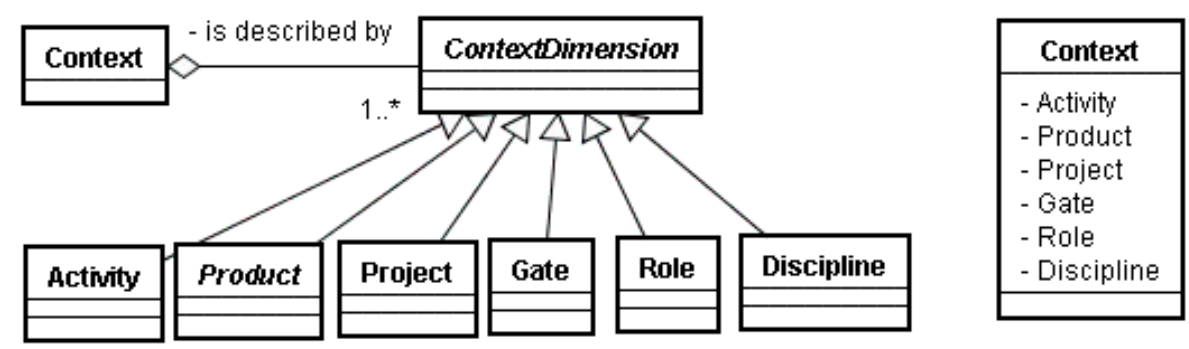

Fig. 3. KEE user context description models

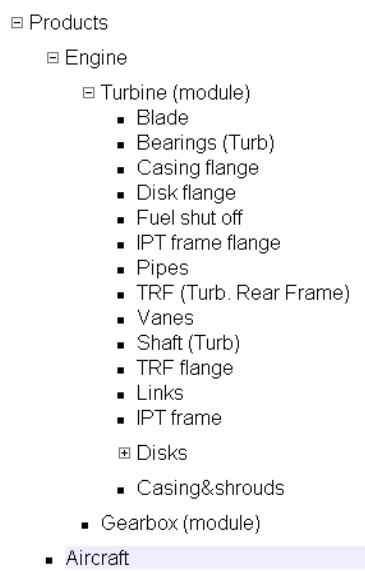

Fig. 4. Tree of possible values for product context dimension

Based on this KEE user context description model, and company context dimensions values, the context of user Daniele working in the AVIO company may be described as follows.

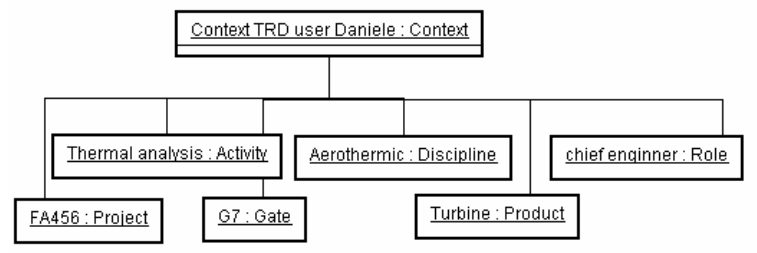

\begin{tabular}{|l|}
\hline Context TRD user Daniele : Context \\
\hline Activity $=$ Thermal analysis \\
Product $=$ Turbine \\
Project $=$ FA456 \\
Gate $=$ G7 \\
Role $=$ Chief engineer \\
Discipline = Aerothermic \\
\hline
\end{tabular}

Fig. 5. Description of context of user Daniele working in the AVIO company 


\section{VIVACE Context Based Search Platform Capabilities}

\subsection{Overview of VIVACE Context Based Search Platform Capabilities}

For the end user, the expected capability of the VIVACE context based search platform is to provide K-El applicable to the user's context described by the following context dimensions: product, activity, project, gate, role and discipline. Therefore, several sub capabilities were developed:

Index K-El on Context. This capability enables the management of the association between a K-El and the different descriptions of contexts in which it was or should be applied.

Context Similarity and K-El Applicability Computation. This capability enables the identification of contexts similar to the user's context, to retrieve K-El which were used in those similar contexts and to compute individual K-El applicability.

At a first glance, these two capabilities may be considered as sufficient for enabling context based search, but since knowledge is not usually indexed on context, the system should include capabilities to learn which knowledge is applicable to which context. Therefore, two new capabilities were developed:

Meta Search in K-Sources. This capability enables searching for K-El in all Ksources through ad-hoc techniques, such as full-text search.

K-EL Applicability Learning and Validation. This capability enables the system to learn that a $\mathrm{K}-\mathrm{El}$ is applicable to a specific context.

These four capabilities enable a learning process which is shown in the following figure:

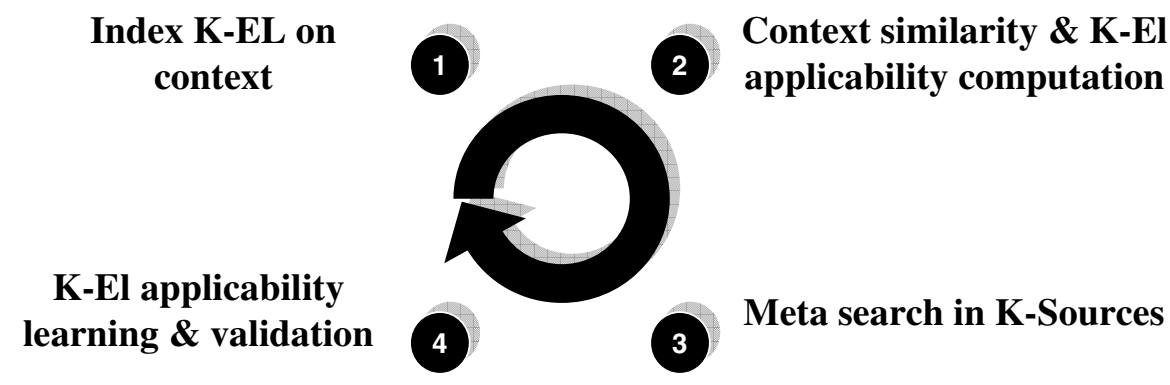

Fig. 6. VIVACE context based search capabilities

The following paragraphs give more detail for each capability

\subsection{Index K-El on Context}

Indexing K-El on context means to say that a specific K-El is applicable to a specific context with a specific level of applicability. The level of applicability could be an input from the expert or more likely a value computed by the platform itself. In order to index knowledge on context, we specified a $K$-El reference and context database 
which enables the management of links between contexts and K-El references. A K$\mathrm{El}$ reference is a pointer to a $\mathrm{K}-\mathrm{El}$, it contains the $\mathrm{K}-\mathrm{El}$ identifier and other information such as a title, a description, a type etc.

The platform includes means to specify and deploy K-Source specific extraction and transformation rules that could be launched on a periodic basis. These rules enable interpretation of $\mathrm{K}-\mathrm{El}$ content in order to generate $\mathrm{K}-\mathrm{El}$ references and possibly to retrieve context information so as to generate the proper links in the $K$ - $E l$ reference and context database.

The conceptual data model of the $K$-El reference and context database is shown in the following figure. This model enables i)to manage many to many relationships with associated applicability between K-El references and contexts, ii)to make the distinction between applicability given by an expert and applicability computed/learnt by the system.

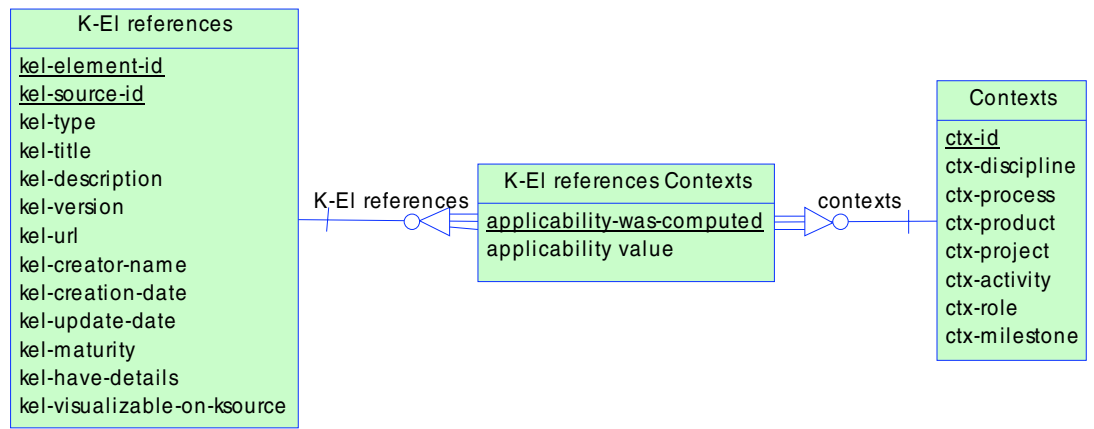

Fig. 7. $K$-El reference and context database conceptual data model

\subsection{Compute Context Similarity and K-El Applicability}

This is the core capability of the platform; it is based on Case Based Reasoning (CBR) technology [10]. In order to develop our CBR application we had to define the case model, the case base, the viewpoints including associated similarity measures and retrieval strategy, and the adaptation strategy.

Case Model. For our CBR application, a case is composed of i) the description of a user context modelled according to KEE user context description model (problem descriptors) ii) the list of $\mathrm{K}-\mathrm{El}$ associated to this context with their applicability (solution descriptors). Problem descriptors are considered as a symbol, an ordered symbol or a taxonomy, as described in the following figure:

\begin{tabular}{|l|}
\hline \multicolumn{1}{|c|}{ Case } \\
\hline Activity: Taxonomy \\
Product : Taxonomy \\
Project : Taxonomy \\
Gate: Ordered Symbol \\
Role: Symbol \\
Discipline: Symbol \\
Applicable K-Elements
\end{tabular}

Fig. 8. Case model 
Case Base. The case base is obtained from the $K$-El reference and context database and it contains the description of all the contexts with associated K-EL.

Viewpoints. Viewpoints contain information about weights and similarity measures to use for each descriptor as well as for selection of the retrieval strategy. For our application, default weights were proposed, similarity measures such as taxonomy and similarity matrices were used and nearest neighbour retrieval method was selected.

Adaptation. The result of the nearest neighbour retrieval is a list of similar contexts with associated K-El and their applicability. Depending on i) the frequency of occurrences of a specific K-El in this list of similar contexts, and ii) the K-El applicability for each similar context; the platform computes individual K-El applicability to user's context.

\subsection{Meta Search in K-Sources}

This capability propagates a full text search request to all K-sources connected to the platform. Then, each K-source relies on its own search capabilities to perform the search (for example Documentum ${ }^{\mathrm{TM}}$ based K-sources which will use Documentum ${ }^{\mathrm{TM}}$ search capabilities and for WWW K-source search will be performed by ad-hoc web search engine such as Google ${ }^{\mathrm{TM}}$. The Results of each K-source search process are then sent back to the platform which aggregates results and possibly adds new K-El reference to the $\mathrm{K}-\mathrm{El}$ reference and context database.

\subsection{Knowledge Applicability Learning and Validation}

Users can assess K-El applicability to his/her context. For K-El that result from fulltext search, applicability is unknown and the user could decide to quantify applicability to his/her context by valuing a percentage from $0 \%$ (not applicable) to $100 \%$ (fully applicable). For K-El that result from contextual search, the user could decide to increase or decrease the applicability value which was computed by the system.

The platform should include a validation process to control the user's feedback process. This validation process may be implemented differently from company to company. For example, some companies may wait for 5 users to give feedback in the same direction to automatically validate, whereas others may ask an administrator to validate manually on a periodic basis.

After validation, the $K$-El reference and context database is updated according to users' feedback thus enriching potential results of any later context based search.

\section{VIVACE Context Based Search Platform Prototype}

\subsection{Overview of Platform Architecture and Implementation}

A first prototype of the platform was developed in 2006 based on a three-layered architecture: 
Portal Layer for enabling a user's context identification and contextual and full text searches. The portal implementation is based on portlet technologies, thus it could be easily integrated into other web applications, such as existing company intranets.

Kernel Layer for enabling context similarity and K-El applicability computation as well as managing the $K$-El reference and context database. The kernel implementation is based on EADS Innovation Works CBR engine.

Knowledge Source Interface (KSI) Layer for enabling extraction and alignment of K-El metadata and multi-source search capabilities. The KSI implementation is based on Documentum ECIS services.

The three different layers were based on open source components (APACHE components and MySQL) and they communicate through web services as shown in the following figure.

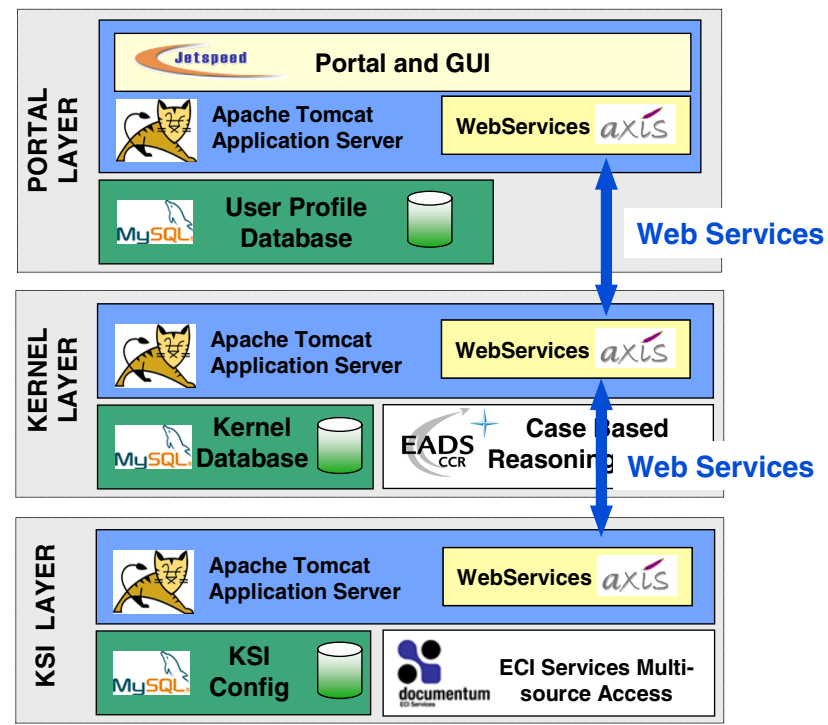

Fig. 9. Platform architecture

\subsection{Platform Prototype User Interface}

The platform prototype user interface is composed of five main panels:

Context Identification Panel. This panel enables the identification of the user's context through setting appropriate context dimensions values.

Search Panel. This panel enables the launch of the contextual search, which may be combined with complex filter-based K-El metadata and full text search in K-sources.

K-El Browser. This panel enables the browsing of search results 


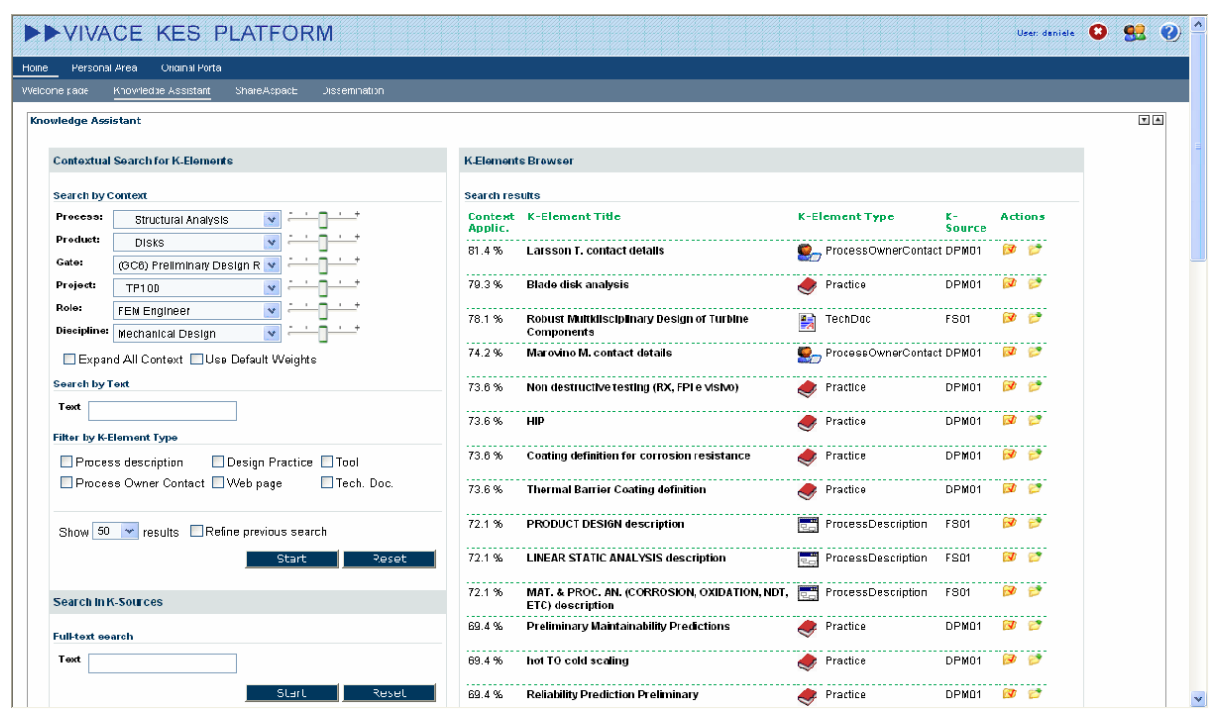

Fig. 10. Search and K-El browser panel

K-El reference and Context Viewer. This panel enables the visualisation of K-El reference and the context in which the $\mathrm{K}-\mathrm{El}$ are applicable.

K-El Applicability Feedback Form. This panel, which is activated each time the user opens a K-El, enables the user to assess the applicability of the K-El with regard to his/her context.

\subsection{Platform Prototype Scenario of Use}

This generic scenario provides a walk through of how we envisage the platform being used.

1. User $\mathrm{A}$ is working in context $\mathrm{Cx}$

2. Platform provides applicable K-El to user A through context based search.

3. User A accesses K-El reference and he/she could decide to open the K-El. For each opened $\mathrm{K}-\mathrm{El}$, the platform requests his/her feedback on K-El applicability.

4. User A is not fully satisfied by K-El obtained in step2, he/she searches for other K-El through a full text search in all K-sources.

5. User A find interesting K-El and he/she records applicability of this K-El to his/her context.

6. User B is working in a context $\mathrm{Cy}$ similar to $\mathrm{Cx}$

7. Platform provides applicable K-El to user B. New K-El which were found in step 4 and said to be applicable by user A in step 5 are automatically provided to user B. 


\subsection{Platform Prototype Experimentation}

Based on the VIVACE Turbine Rotor Design (TRD) use case, successful experimentations were conducted by KEE partners to validate the prototype. AVIO experts were involved in the project, they participated in the specification of the TRD context description model and they provided knowledge about similarities between context dimensions values. AVIO internal K-sources and external K-sources, such as the World Wide Web, were connected to the platform.

Results of these first experimentations validate that the platform can help provide the user with applicable knowledge depending on his/her context. Furthermore, promising results were obtained for the indexing of web pages based on context. The platform promotes collective learning about which information available on the web is applicable to which context. Thus, the platform offers promising capabilities to solve the information overload issue that users encounter in engineering activities.

\section{Perspectives}

\subsection{Future Work}

Piloting Activities. A piloting phase is scheduled in early 2007 in order to validate the platform in a real industrial environment and to measure associated benefits. The platform will be used by an operational team working on Turbine Rotor Design at AVIO.

SWOT analysis. SWOT analysis aims at evaluating the Strengths, Weaknesses, Opportunities and Threats involved in our proposal. This analysis will rely on consolidated results obtained from the piloting activities and analysis of other proposals for enabling context based search such as for example, those proposed by Kirsh-Pinheiro et al.[11] and David Leake et al.[12].

Context Modelling Enhancement. As described in part III, we based our work on a simplified context description model. The objective is to enhance this context description model in order to take into account i) richer information about existing context dimension (eg context dimension classes rather than attributes, for example, the discipline context dimension class may be described by several attributes such as the name of discipline, the level of expertise, etc.) ii)new context dimension, for example to better describe user profile and tool used iii)latest research results on context modelling.

Context Similarity Computation. The objective is to refine the context similarity computation algorithm to cope with the enhanced context description model and to better exploit existing links between context dimensions in the engineering domain model.

Guidelines Elaboration. As software platform or tool alone will never be an answer to a knowledge management issue, appropriate guidelines focusing on organizational, methodological and behavioural aspects should be elaborated. These guidelines will be used together with the platform to address the challenge of in-context knowledge delivery. 
Integration Activities. The platform will be integrated with other advanced capabilities developed in the VIVACE project, such as Engineering Data management [13] (EDM) and Design To Decision Objectives (DTDO). The objective is to provide in-context delivery capabilities for knowledge as well as for product and simulation data. All results should be integrated in the VIVACE toolbox which will be a collaborative engineering environment that will notably raise the level of support for efficient decision making in engineering.

\subsection{Research Perspectives}

Advanced Context Identification. The objective is to enable automatic or assisted identification of user context. A user's context may be identified through monitoring and analysis of user behaviours, application and data used etc. However, it may not be possible to identify all context dimensions automatically, so the user may still have to set some context dimension values and validate the identified ones.

Knowledge Pushing. The objective is to combine advanced context identification capabilities and context based search capabilities to automatically push applicable knowledge to the user depending on his/her context. In other words, the aim is to develop a pro-active search system that does not necessarily require the user to take the search initiative.

Learning Enterprise. The objectives are twofold: at the software level to enhance and develop users' feedback mechanisms in our platform; at the organizational level to promote a learning culture in which users are eager to provide their feedback for the benefit of others. Nowadays, with participative tools associated to the Web2.0 framework, an efficient learning organization emerges on the web. Enabling this transformation in industry is still a challenge.

\section{Conclusion}

In order to face the new competitive situation in industrial companies, the design cycle must be shortened and engineers are asked to design right first time. On the one hand, shortening the design cycle leaves less time for the engineer to search for knowledge, on the other hand, the requirement to design right first time increases the need for knowledge search and reuse. The key issue is then to provide the right knowledge to the right user at the right time in the design process.

In order to face this issue we proposed a context based search platform that enables in-context delivery of knowledge. First results of platform experimentation are very promising. The platform enables collective learning of which knowledge is applicable to which context and efficient searching for knowledge applicable to the user context.

We believe that this context based search platform is a first step toward the development of pro-active search systems. Therefore further research work is required in order to develop automatic context identification capabilities and to use them together with context based search capabilities. Nowadays, engineers are often not even aware that there may be some knowledge available to help them, so they do not take the search initiative. For this reason, pro-active search systems that may push 
applicable knowledge without requiring user initiative are seen as the ultimate answer for supporting engineering activities.

Acknowledgements. The VIVACE integrated project is partly sponsored by the Sixth Framework Programme of the European Community (2002-2006) under priority 4 "Aeronautics and Space" as integrated project AIP3 CT-2003-502917. VIVACE context based search platform was developed by AVIO, EADS IW and Xerox.

\section{References}

1. Value Improvement through a Virtual Aeronautical Collaborative Entreprise.: www.vivaceproject.com

2. Schreiber, G., Akkermans, H., Anjewierden, A., de Hoog, R., Shadbolt, N., Van de Velde, W., Wielinga, B.: Knowledge Engineering and Management: the CommonKADS Methodology. MIT Press, Cambridge (2000)

3. Callot, M., Oldham, K., Stokes, M., Godwin, N., Brimble, R., Klein, R., Sellini, F., Merceron, F., Danino, D.: MOKA - Methodology and Tools Oriented to Knowledge based Engineering. Project EP25418 Public report $\mathrm{N}^{\circ} 2$ (1999)

4. Bazire, M., Brézillon, P.: Understanding Context Before Using It. In: Dey, A.K., Kokinov, B., Leake, D.B., Turner, R. (eds.) CONTEXT 2005. LNCS (LNAI), vol. 3554, Springer, Heidelberg (2005)

5. Dey, A.K, Abowd, G.D.: Toward a Better Understanding of Context and Context Awareness (1999)

6. Lelouma, T., layaïda N.: Context Aware Adaptation for Mobile Devices (2004)

7. Kirsch-Pinheiro, M., Villanova-Olivier, M., Gensel, J., Martin, H.: Representing Context for an Adaptive Awareness Mechanism. In: de Vreede, G.-J., Guerrero, L.A., Marín Raventós, G. (eds.) CRIWG 2004. LNCS, vol. 3198, Springer, Heidelberg (2004)

8. Edmonds, B.: The Pragmatic Roots of Context (1999)

9. Longueville, B., Gardoni, M.: A Survey of Context Modeling: Approaches, Theories and Use for Engineering Design Researches. In: Blackburn, P., Ghidini, C., Turner, R.M., Giunchiglia, F. (eds.) CONTEXT 2003. LNCS, vol. 2680, Springer, Heidelberg (2003)

10. Watson, I., Marir, F.: Case based Reasoning: a review. The knowledge Engineering review (1994)

11. Kirsch-Pinheiro, M., Villanova-Olivier, M., Gensel, J., Martin, H.: Context-aware Filtering for Collaborative Web Systems: Adapting the Awareness Information to the User's Context. In: Preneel, B., Tavares, S. (eds.) SAC 2005. LNCS, vol. 3897, Springer, Heidelberg (2006)

12. Leake, D., Maguitman, A., Reichherzer, T.: Exploiting Rich Context: An incremental Approach to Context-Based Web Search. In: Blackburn, P., Ghidini, C., Turner, R.M., Giunchiglia, F. (eds.) CONTEXT 2003. LNCS, vol. 2680, Springer, Heidelberg (2003)

13. Nguyen, V.T., Feru, F., Guellec, P., Yannou, B.: Engineering Data Management for extended enterprise - Context of the European VIVACE Project. In: PLM06 International Conference (2006) 